\title{
Online precision gas evaluation of the ATLAS Muon Spectrometer during LHC Run1
}

\author{
Cong Geng \\ Department of Physics, The University of Michigan, Ann Arbor, MI 48109-1120, USA \\ Department of Modern Physics, University of Science and Technology of China, Hefei 230026, China
}

(On behalf of the ATLAS Muon Collaboration)

\begin{abstract}
The ATLAS Muon Spectrometer, a six story structure embedded in a toroidal magnetic field, is constructed of nearly 1200 Monitored Drift Tube chambers (MDTs) containing 354,000 aluminum drift tubes. The operating gas is 93\% Ar + $7 \% \mathrm{CO}_{2}$ with a small amount of water vapor at a pressure of 3 bar. The momentum resolution required for ATLAS physics demands that MDT gas quality and the associated gas dependent calibrations be determined with a rapid feedback cycle. During the LHC Run1, more than 2 billion liters of gas flowed through the detector at a rate $100,000 \mathrm{l} / \mathrm{hr}$. Online evaluation of MDT gas in real time and the associated contribution to the determination of the time-to-space functions was conducted by the dedicated Gas Monitor Chamber (GMC). We report on the operation and results of the GMC over the first three years of $\mathrm{LHC}$ running. During this period, the GMC has operated with a nearly $100 \%$ duty cycle, providing hourly measurements of the MDT drift times with $1 \mathrm{~ns}$ precision, corresponding to minute changes in gas composition.
\end{abstract}

\section{INTRODUCTION}

$\mathbf{I}$ $\mathrm{N}$ this paper we describe the performance of Gas Monitor System dedicated to the continuous gas monitoring and drift time calibration of the precision Monitored Drift Tube (MDT) chambers [1] in the ATLAS Muon Spectrometer [2]. The drift tube is the fundamental sensitive element in the MDT chambers. Nearly 1200 MDT chambers incorporated in the Muon Spectrometer consist of approximately 354,000 MDT tubes, which are constructed from $3 \mathrm{~cm}$ diameter extruded aluminum, pressurized to 3 bar with a $93 \% \mathrm{Ar}$ and $7 \% \mathrm{CO}_{2}$ gas mixture, and operated at $3080 \mathrm{~V}$ to establish a gain of 20,000 .

The spectrometer's precision coordinate is transverse to the tube axis and is determined by the radial distance of closest approach to the anode wire of a charged particle passing through a drift tube. Ionization electrons drift towards the sense wire and initiate an avalanche producing an anode wire signal. Conversion of the drift time to a radius is made with time-to-space relations known as RT functions. Fig. 1 shows the sketch of the drift tube and a muon passing through it and ionizing the gas.

The spectrometer is designed to achieve momentum resolution of $3 \%$ at $100 \mathrm{GeV}$ and $10 \%$ at $1 \mathrm{TeV}$ over a rapidity interval of $-2.7<\eta<2.7$. The momentum resolution is based on a single drift tube intrinsic spatial resolution of $80 \mu \mathrm{m}$. To attain this resolution for such a large detector and to do so continuously over extended periods of many months requires high precision construction, a continuous

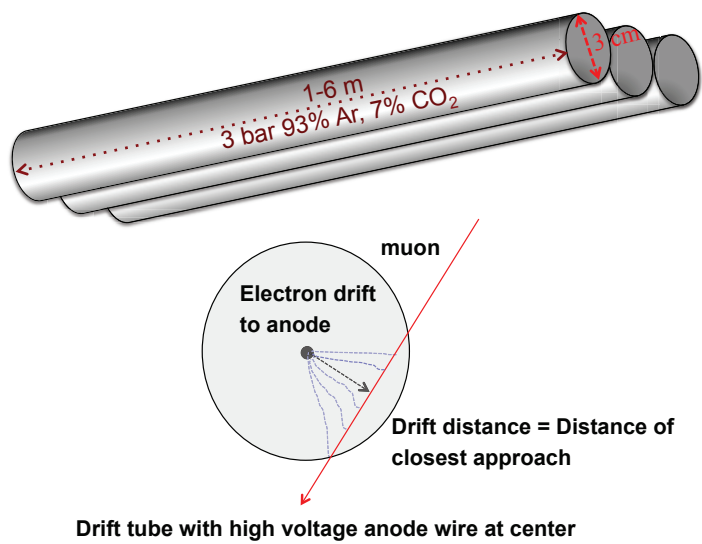

Fig. 1: Sketch of the drift tube and a muon passing through it and ionizing the gas.

alignment system and very careful monitoring of the gas quality. Therefore, monitoring of the overall condition of the MDT gas mixture is an important component to the calibration program.

A mini-chamber of MDTs provides continuous and online monitoring of the gas quality. It is constructed to sample gas from the main MDT supply and return gas trunk lines. A pair of scintillators provides a cosmic ray trigger. An automated, sequenced running of data acquisition and offline analysis records the cosmic ray data and processes the drift time spectra. The analysis output is represented by sets of fit parameters that characterize the gas for well-measured temperature, pressure, and zero magnetic field. An important analysis parameter most sensitive to the gas mixture is the maximum drift time, $T_{\max }$.

\section{Gas Monitor System}

The gas monitor system shown in Fig. 2 contains 96 tubes with dimensions $50 \mathrm{~cm} \times 70 \mathrm{~cm} \times 21 \mathrm{~cm}$. While they are significantly shorter than any ATLAS Muon Spectrometer MDTs, the drift tubes of GMC mirror the performance of conventional ones because the manufacture and assembly processes are same.

The 96 tubes are grouped into two identical multilayers, each of which consists of a vertical pair of $3 \times 8$ sections. 


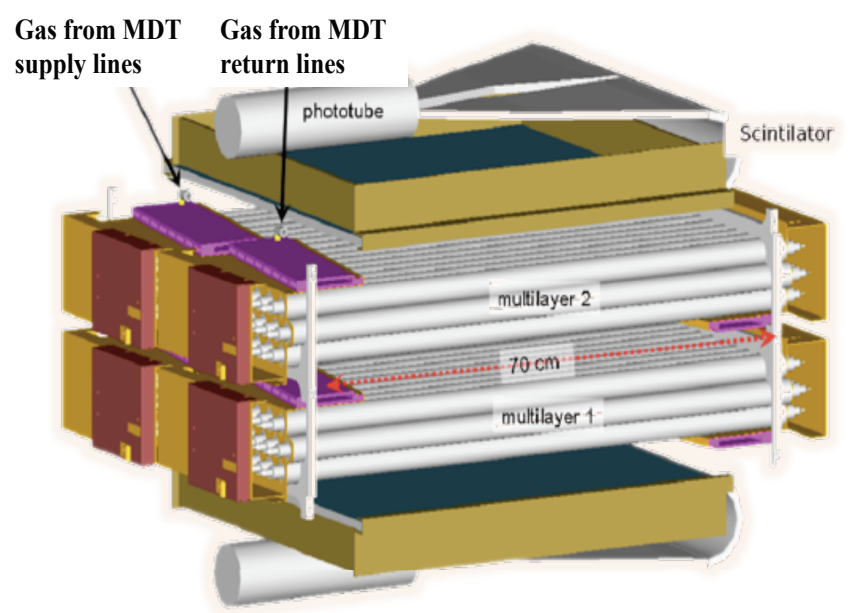

Fig. 2: Drawing of the GMC.

These two chamber partitions are defined by independent gas systems. In this fashion one partition can sample the input gas stream to the Muon Spectrometer while the neighboring partition samples from the return gas lines. These two partitions enable the chamber to track gas quality and measure differential performance simultaneously.

The GMC acquires data from cosmic ray triggers, which consist of a pair of plastic scintillators. These scintillators are positioned horizontally, immediately above and below the chamber, as indicated in Fig. 2.

The drift time spectrum is the key tool to gauge drift tube performance. The spectra of all tubes in a partition are combined. The summing of the spectra is facilitated by the fact that each tube is subject to essentially identical operating conditions. The typical drift spectrum is depicted in Fig. 3, where there are two evident edges for rising and trailing. For the purpose of tracking the MDT gas properties, an operational $T_{\max }$ is defined by fitting the rising and trailing edges of the drift spectrum. These fits use modified Fermi-Dirac functions:

$$
f(t)=\frac{P_{1}+P_{4} t}{1+e^{\left(P_{2}-t\right) / P_{3}}} .
$$

The 50\% point of the rising or trailing is $P_{2}$. The rising edge parameter $P_{2}$ is referred to as $T_{0}$ corresponding to tracks passing at the anode wire, and the trailing edge parameter $P_{2}$ corresponding to tracks passing at the tube wall. The difference of the parameters corresponding to 50\% rising/trailing edge defines $T_{\max }$ :

$$
T_{\text {max }}=B_{\text {trailing }}-B_{\text {rising }} .
$$

The temperature of the GMC is monitored in eight locations and is used, along with the pressure sensor readout, to establish the precise operating conditions, and to enable temperature and pressure corrections to the drift time. The standard conditions are chosen to conform to the ATLAS MDT's located in the underground cavern, which is the reference for GMC corrections. So, final measured $T_{\max }$ is very sensitive to the change in gas composition, which are computed using Garfield

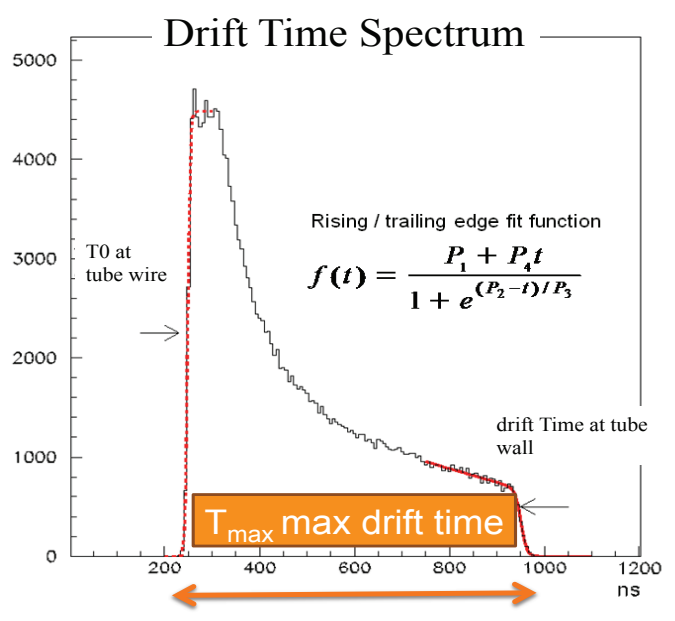

Fig. 3: Drift spectrum for one partition of the GMC.
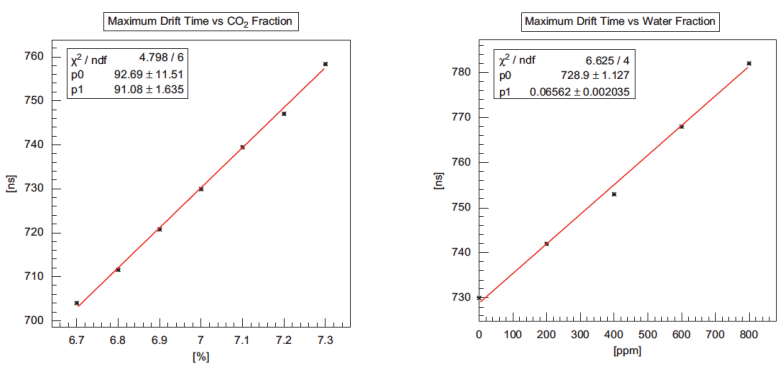

Fig. 4: Distributions of $T_{\max }$ as functions of $\mathrm{CO}_{2}$ and water vapor fractions respectively.

and shown in Fig. 4 (distributions of $T_{\max }$ as functions of $\mathrm{CO}_{2}$ and water vapor fractions respectively).

\section{GMC RESULTS FOR LHC RUN1}

Fig. 5a shows the ratio of $\mathrm{CO}_{2}$ fraction to $\mathrm{Ar}$ during 2012 LHC running, which is calculated with the data from the detector control system (DCS) and indicates the fraction of $\mathrm{CO}_{2} / \mathrm{Ar}$ is quite stable. However, the ambient humidity increased by about $10 \%$ from spring to summer, as shown in Fig. 5b (b), which could result in the water vapor fraction in the tube varying accordingly.

During the 2010 - 2012 three year Run1 period of LHC, the GMC was working very well and monitoring the gas system continuously. Fig. 6 shows the recorded $T_{\max }$ for the years 2010, 2011, and 2012, respectively. The $T_{\max }$ varies by a few ns to a maximum of $10 \mathrm{~ns}$ in each year, and the variation is within about $1 \mathrm{~ns}$ per month. This variation is associated with the seasonal change in ambient humidity. Fig. 7 reflects the $T_{\max }$ difference between supply and return gas flow retrieved from 2012 data, where the distribution is also quite stable as function of time and the difference is around $0.5 \mathrm{~ns}$ through the whole year. This indicates that the overall gas composition variations throughout the MDT system are negligible. 


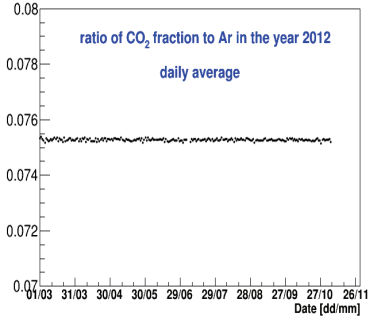

(a)

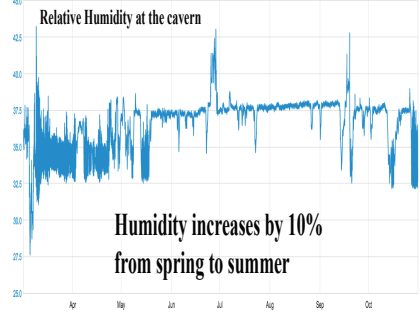

(b)
Fig. 5: The fraction of $\mathrm{CO}_{2} / \mathrm{Ar}$ (a) and ambient humidity (b) as function of time in 2012 .

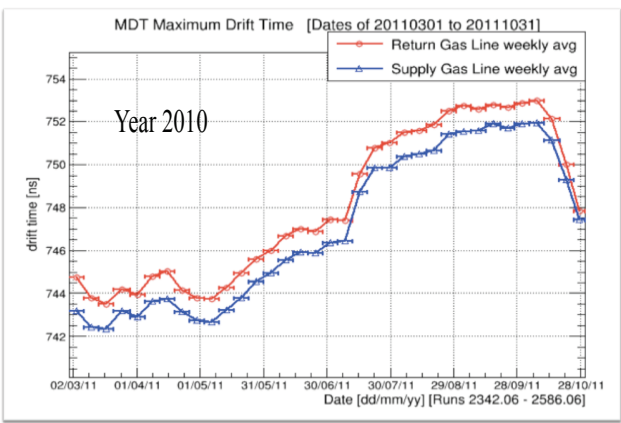

(a)

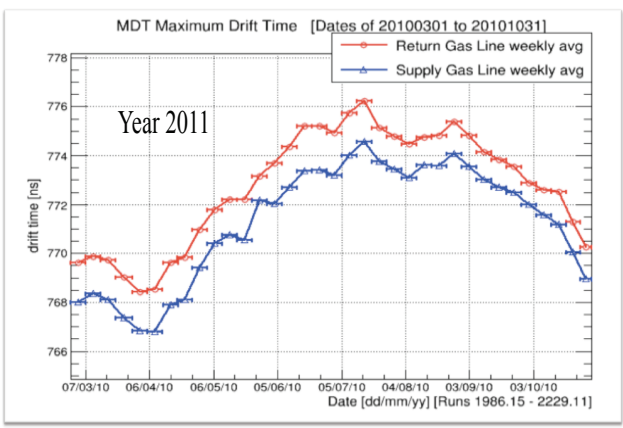

(b)

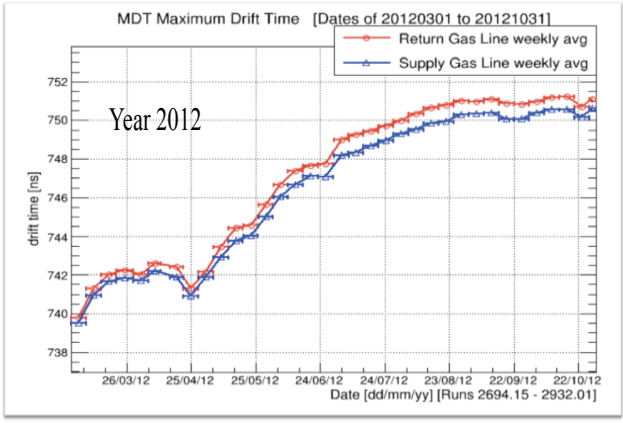

(c)

Fig. 6: Maximum drift time distributions recorded by GMC for the year 2010, 2011, and 2012.

\section{SUMmARY}

The gas monitor system described in the paper has the unique capability to acquire and analyze the ATLAS Muon Spectrometer MDT drift time spectra quickly and accurately.

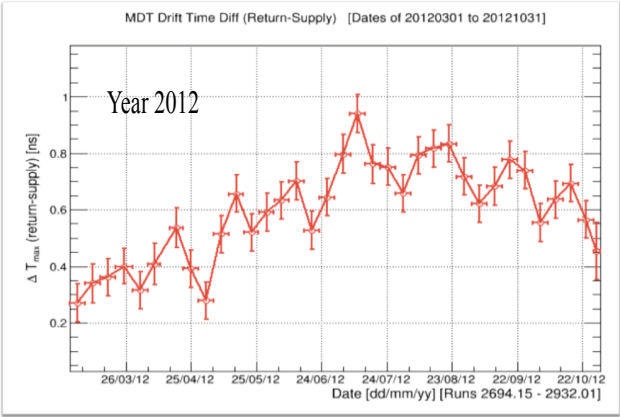

Fig. 7: Distribution of $T_{\max }$ difference between supply and return gas flow.

The supply and return gas flow are evaluated simultaneously. Using cosmic ray muon data, the GMC produces drift spectra hourly and reports the important $T_{\max }$, which is most sensitive to the gas composition, pressure, and temperature. The pressure and temperature have been corrected to nominal values, so the value of $T_{\max }$ can flag spontaneous gas system faults. According to the distributions recorded in the period of LHC Run1, years 2010, 2011, and 2012, we did not observe any serious faults and the GMC worked stably. The variation of $T_{\max }$ is less than $10 \mathrm{~ns}$. For each month, $T_{\max }$ only varied by about $1 \mathrm{~ns}$. The variations in $T_{\max }$ are consistent with the variations in water vapor fraction less than $\pm 200 \mathrm{ppm}$ per year and were addressed by providing new calibrations for the affected periods.

A further application of this system [3] is to produce a standard RT function specific to well measured gas temperature, gas pressure and to the actual MDT gas used in the underground ATLAS MDT chambers.

\section{ACKNOWLEDGMENT}

We acknowledge the contributions of the ATLAS Muon Spectrometer Calibration group. This work is funded in part by the U.S. Department of Energy.

\section{REFERENCES}

[1] D. Levin et al., Drift time spectrum and gas monitoring in the ATLAS Muon Spectrometer precision chambers, Nucl. Instrum. Meth. A588 (2008) 347-358.

[2] ATLAS Collaboration, The ATLAS Experiment at the CERN Large Hadron Collider, JINST 3 (2008) S08003

[3] N. Amram et al., Streamlined Calibrations of the ATLAS Precision Muon Chambers for Initial LHC Running, Nucl. Instrum. Meth. A671 (2012) 40-50 